\title{
Numerical investigations on the performance of external-cavity mode-locked semiconductor lasers
}

\author{
Josep Mulet ${ }^{a, b}$ and Jesper Moerk ${ }^{a}$ \\ ${ }^{a}$ Research Center COM, Technical University of Denmark, Building 345V, \\ DK-2800 Kgs. Lyngby (Denmark) \\ ${ }^{b}$ Departament de Física, Universitat de les Illes Balears, \\ E-07122 Palma de Mallorca (Spain)
}

\begin{abstract}
The performance of an external-cavity mode-locked semiconductor laser is analyzed theoretically and numerically. Passive mode-locking is described using a fully-distributed time-domain model including fast effects, spectral hole burning and carrier heating. We provide optimization rules in order to improve the mode-locking performance, such as reducing the pulsewidth and time-bandwidth product as much as possible. Timing jitter is determined by means of extensive numerical simulations of the model, demonstrating that an external modulation is required in order to maintain moderate timing-jitter and phase-noise levels at low frequencies. The effect of the driving conditions is investigated in order to achieve short pulses and low timing jitter. Our results are in qualitative agreement with reported experiments and predictions obtained from the master equation for mode-locking.
\end{abstract}

Keywords: Semiconductor laser, Mode-locking, Timing jitter, Short pulse generation

\section{INTRODUCTION}

Mode-locked semiconductor lasers are a flexible source of intense short optical pulses. ${ }^{1-3}$ The viability of these devices in high-speed communication systems using optical time-division multiplexing (OTDM) relies on the possibility of achieving short pulsewidths and low timing jitter levels. For example, transmission at $640 \mathrm{Gbit} / \mathrm{s}$ imposes hard requirements, namely, pulsewidths $\sim 0.4 \mathrm{ps}$ and timing jitter less than $100 \mathrm{fs}$. To date, it is still unclear what the ultimate performance of mode-locked semiconductor lasers is. In particular, it is important to understand the fundamental limitations to the minimum attainable pulsewidth. Also, the stability of such a pulsed source against noise is a question of crucial interest.

From the modelling point of view, pulse formation and noise properties of mode-locked semiconductor lasers are challenging problems, since they involve the interplay of several mechanisms including, dispersion, self-phase modulation, nonlinear dynamics, and amplified spontaneous emission (ASE). Mode-locking in semiconductor lasers has been described by means of lumped-element models, partially-integrated models, fully-distributed time-domain models, and frequency domain models. Passive mode-locking using saturable absorbers is most conveniently analyzed in the time domain, although a many-mode interacting framework has been recently established. ${ }^{4}$ The master equation for mode-locking (MEML) describes the lumped effect by any one of the mechanisms acting on the pulse per roundtrip. ${ }^{5-9}$ The MEML has been extensively used in the literature owing to its simplicity and the possibility of obtaining analytical predictions for the pulse shape, stability and attainable pulsewidths. In addition the perturbation theory of the MEML, established in references, ${ }^{9,10}$ describes the evolution of four orthogonal fluctuations driven by spontaneous emission, namely, timing, emission frequency, pulse energy and carrier density fluctuations, that in turn fully determine the pulse jitter. In spite of these achievements, there exists a number of effects that are difficult to treat using the MEML, suggesting that more general descriptions are required. Some of these effects include, i) origin of the pulse train and its instabilities,

Further author information: (Send correspondence to J. Mulet)

J. Mulet: E-mail: mulet@imedea.uib.es, Telephone: +34 971 172536, Fax: +34 971173426

J. Moerk: E-mail: jm@com.dtu.dk, Telephone: +45 4525 5765, Fax: +45 45936581

1 Proc. SPIE 5452-70 (2004) 
ii) synchronization locking, iii) colliding effects, iv) material dynamics occurring at pulsewidth time scales, and v) timing fluctuations induced due to gain depletion ${ }^{11}$ and carrier-induced changes in group velocity.

The aim of this paper is to develop a detailed theoretical description for mode-locked semiconductor lasers that enables a direct comparison between theory and experiments. Among the different possible descriptions, we use a fully-distributed time-domain model describing the optical propagation within the entire device by including gain nonlinearities, bandwidth limitation and spontaneous emission. This approach offers several advantages when comparing with the MEML. For instance, it is relatively simple to account for the effects i)-v) discussed above and allows for describing a variety of configurations. Here we investigate the dynamical properties of an externalcavity mode-locked semiconductor laser (ECMLL), where the absorber is placed in a self-colliding configuration. The ECMLL offers the possibility of tuning the repetition rate and emission wavelength in intervals much larger than monolithic constructions. The interest in ECMLL is also motivated by the relatively short pulsewidths and timing jitter levels that have been demonstrated. ${ }^{1}$ In section 2 we describe our model for passive mode-locking. In Sec. 3, we use the model to analyze the performance of a $10 \mathrm{GHz}$ repetition rate device. The evolution of the pulsewidth and timing jitter is investigated when varying the injection current and the reverse bias in the saturable absorber. In Sec. 4, we analyze the timing jitter for the case of passive mode-locking, and hybrid mode-locking that is introduced to reduce the low-frequency jitter. We put special emphasis in explaining the numerical method used to determine timing jitter. Finally, Sec. 5 is devoted to concluding and summarizing our paper.

\section{THE MODEL}

The ECMLL, as shown in Fig. 1, is composed by a two section laser diode and a diffraction grating as external mirror. The electric field within the laser diode is expressed as the superposition of two counter-propagating waves $E^{ \pm}(z, t)$. The evolution of the two counter-propagating waves is described by the travelling wave model

$$
\begin{aligned}
\pm \frac{\partial E^{ \pm}(z, t)}{\partial z} & +\frac{1}{v_{g, b}} \frac{\partial E^{ \pm}(z, t)}{\partial t}=\frac{1}{2}\left[\Gamma\left(g\left(\omega_{0}, \zeta\right)-i \frac{\omega_{0}}{c} \Delta n\left(\omega_{0}, \zeta\right)\right)-\alpha_{i n t}\right] E^{ \pm}(z, t) \\
& -\frac{\Gamma}{2}\left(\frac{\partial}{\partial \omega}\left[\frac{\omega}{c} \Delta n(\omega, \zeta)\right]_{\omega_{0}}+i\left[\frac{\partial g(\omega, \zeta)}{\partial \omega}\right]_{\omega_{0}}\right) \frac{\partial E^{ \pm}(z, t)}{\partial t} \\
& +\left(i \frac{\beta_{2, b}}{2}+i \frac{\Gamma}{4} \frac{\partial^{2}}{\partial \omega^{2}}\left[\frac{\omega}{c} \Delta n(\omega, \zeta)\right]_{\omega_{0}}-\frac{\Gamma}{4}\left[\frac{\partial^{2} g(\omega, \zeta)}{\partial \omega^{2}}\right]_{\omega_{0}}\right) \frac{\partial^{2} E^{ \pm}(z, t)}{\partial t^{2}}+F^{ \pm}(z, t)
\end{aligned}
$$

The slowly-varying envelope of the electric field is taken with respect to the optical carrier frequency $\omega_{0}$. The group velocity $(\mathrm{GV})$ and group-velocity dispersion (GVD) arising from the background refractive index are $v_{g, b}$ and $\beta_{2, b}$ respectively. $\Gamma$ is the optical confinement factor that scales with the number of quantum wells (QWs), and $\alpha_{i n t}$ the internal losses. The material gain and carrier-induced refractive index are denoted by $g$ and $\Delta n$ respectively. We use the variable $\zeta=\left\{N_{q w}, n_{c}, n_{v}, T_{c}, T_{v}, V_{a b s}\right\}$ to describe the functional dependence of the

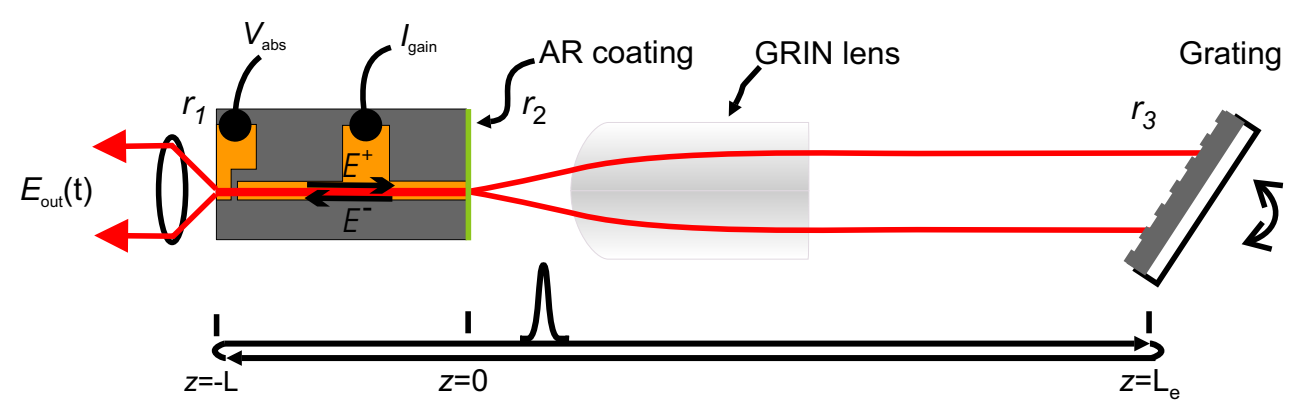

Figure 1. Sketch of the ECMLL as described in reference. ${ }^{12}$ 
gain and index of refraction on, carrier densities, plasma temperature, absorber voltage, etc. We recall that each of these dependencies evolve at different characteristic time scales. The second and third terms on the rhs of Eq. (1) describe, up to second order, the gain and refractive index dispersion induced by the QWs. Finally $F^{ \pm}(z, t)$ are Langevin noise sources that describe spontaneous emission processes. These sources have zero mean and correlation $^{13}$

$$
\left\langle F^{ \pm}(t, z) F^{ \pm *}\left(t^{\prime}, z^{\prime}\right)\right\rangle=\frac{n_{s p} \Gamma g\left(\omega_{0}, \zeta\right)}{V_{g}} \delta\left(t-t^{\prime}\right) \delta\left(z-z^{\prime}\right),
$$

$V_{g}$ being the volume of the active region in the gain section, $n_{s p}=N_{q w} /\left(N_{q w}-\mathcal{N}_{t}\right)$ the inversion factor, and $\mathcal{N}_{t}$ the carrier density at transparency. The level of spontaneous emission is thus determined by the needed cavity gain and by the degree of inversion.

We consider the geometry shown in Fig. 1, i.e., the left output facet is placed at $z=-L$, the right laser facet at $z=0$, and the grating position is $z=L_{e}$. The counter-propagating waves fulfill the following boundary conditions

$$
\begin{aligned}
E^{+}(z=-L, t) & =r_{1} E^{-}(z=-L, t), \\
E^{-}(z=0, t) & =r_{2} E^{+}(z=0, t)+R(t),
\end{aligned}
$$

$R(t)$ being the filtered time-delayed feedback from the diffraction grating, whereas the imperfect AR coating $r_{2}$ is responsible for multiple internal roundtrips. If we consider a Gaussian filtering function and only one external reflection, the feedback term can be written (in time domain) as the convolution of the filter with the delayed field leaving the laser a time $\tau_{e}=2 L_{e} / c$ earlier

$$
R(t) \approx\left(1-r_{2}^{2}\right) r_{3} \hat{\xi} \frac{\Delta \omega_{B}}{\sqrt{4 \pi\left(1-i \alpha_{B}\right)}} \int_{-\tau_{e}}^{\infty} d T e^{-T \Delta \omega_{B}\left[i \Delta_{B}+\frac{T \Delta \omega_{B}}{4\left(1-i \alpha_{B}\right)}\right]} E^{+}\left(z=0, t-\tau_{e}-T\right) .
$$

The grating bandwidth is $\Delta \omega_{B}$, the relative detuning $\Delta_{B}=\left(\omega_{B}-\Omega\right) / \Delta \omega_{B}$, and the dispersion of the grating $D_{\text {grat }}=2 \alpha_{B} / \Delta \omega_{B}^{2} . r_{3}$ is the grating (field) reflectivity, $r_{2}$ is the AR-coating reflectivity, and $\hat{\xi}$ is the coupling efficiency of the external cavity.

The gain model accounts for fast relaxation of the carrier distributions towards quasi-equilibrium Fermi-Dirac distributions due to spectral hole burning and carrier-heating. For a detailed description of the material model the reader is addressed to the references. ${ }^{14-16}$ The carrier-induced refractive index $\Delta n(\omega, \zeta)$ is computed from the material gain using Kramers-Krönig relations ${ }^{17}$ and neglecting fast effects. The absorber model is similar to the gain model, except for including bandgap renormalization with carrier-density and reverse bias (Quantumconfined Stark effect). ${ }^{16,18}$ The absorber recovery time decreases with the reverse bias as found experimentally ${ }^{19}$; $\tau_{a b s}\left(V_{a b s}\right)=\tau_{0} \exp \left(V_{a b s} / V_{\text {ref }}\right)$ with $\tau_{0}=22 \mathrm{ps}$, and $V_{\text {ref }}=2 \mathrm{~V}$. In order to obtain a better agreement with the experimental dependencies, the polarization dephasing rate in the absorber is slightly increased with the reverse bias. $^{20}$

We use a uniform computational mesh to integrate Eq. (1) with $\Delta z=v_{g, b} \Delta t$. The electric fields are propagated over the characteristic lines neglecting dispersion terms. Dispersion is applied in a second step by discretizing the temporal derivatives in an explicit way. Boundary conditions are applied at the edges of the laser diode and the convolution integral (5) is implemented as a digital filter. Finally, the material variables are updated using a fourth order Runge-Kutta method. White Gaussian random numbers are generated using the numerical inversion method developed in reference. ${ }^{21}$

\section{DEVICE PERFORMANCE}

In this section, we analyze the performance of an ECMLL by numerical simulations of the model outlined above, from which we infer some optimization rules. It is known that the maximum attainable bit rate, in a OTDM system, is governed by the pulsewidth and timing jitter levels as well as the appearance of dynamical instabilities. We investigate the evolution of these quantities with respect to the variation of some experimentally accessible control parameters. For the sake of clarity, the evolution of timing jitter shall be presented in the next section. 

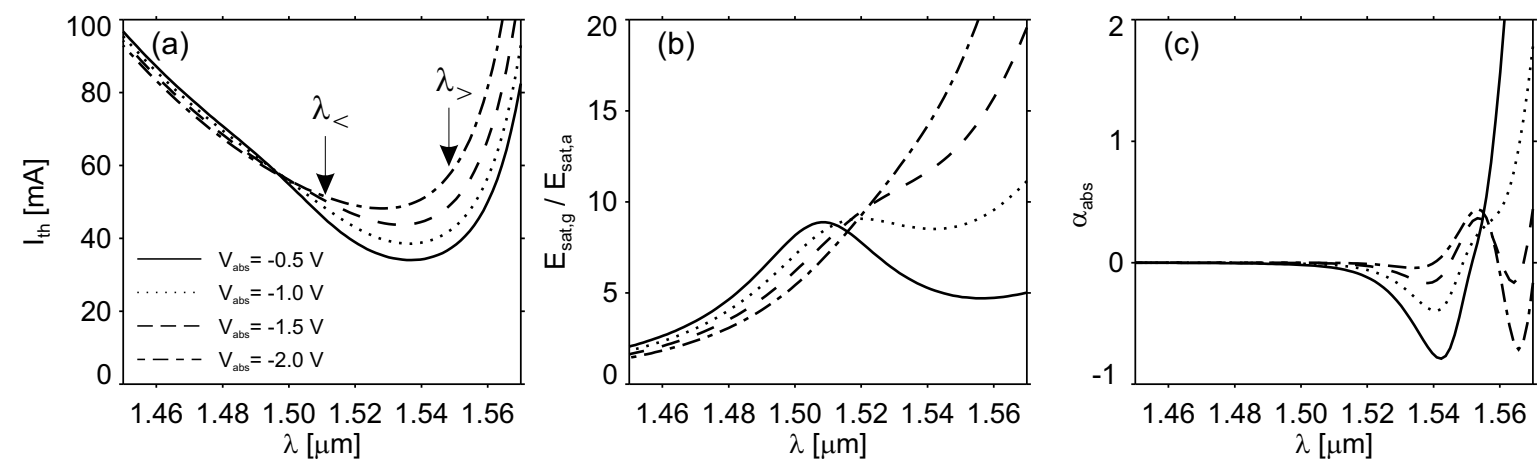

Figure 2. Several mode-locking quantities computed as function of the emission wavelength using the gain model. (a) Threshold current, (b) ratio of saturation energies in the gain and absorber sections, and (c) alpha-factor in the absorber (unsaturated). $\lambda_{>,<}$stand for two different working wavelengths.

As an example, we use our model to simulate a $10 \mathrm{GHz}$ repetition rate ECMLL with a $550 \mu \mathrm{m}$ long amplifier, $30 \mu \mathrm{m}$ reverse-biased absorber, and a $20 \mu \mathrm{m}$ transition region. ${ }^{12,22}$ A long laser chip is preferred because of the reduced magnitude of the trailing pulses originated by an imperfect AR-coating. For a residual power reflectivity of the AR-coating of $10^{-5}$, we obtain trailing pulses with extinction ratio of the order of $\sim 30 \mathrm{~dB}$ whereas only $\sim 20 \mathrm{~dB}$ is achieved in a $300 \mu \mathrm{m}$ long device in agreement with experimental observations. ${ }^{12,23}$ It is also found that the extinction ratio improves with the reverse bias due to the faster recovery of the absorption.

We use the material model to determine the evolution of the travelling waves, but also to find the variation of some mode-locking parameters with the operation conditions. In Fig. 2, the threshold current, the ratio of saturation energies $s$, and alpha Henry factor in the absorber are calculated as function of the emission wavelength and reverse bias. The minimum in threshold current corresponds to the alignment of the gain peak with the central wavelength of the grating. We can also observe a steeper increase in threshold current at longer wavelengths, reflecting the smaller differential gain on the red-side of the gain spectrum. The threshold current significantly increases with the reverse bias, indicating that the internal and mirror losses are moderate. The ratio of saturation energies enters into the pulsewidth determination, ${ }^{8}$ whereas the sign of the pulse chirp depends on the sign of the alpha-factor in the absorber. ${ }^{24}$

\subsection{Stability and pulsewidth}

The injection current and reverse bias are varied in order to generate the phase diagrams. An example of such a phase diagram is shown in Fig. 3(a). We can identify three well differentiated regions: below threshold with no laser radiation (NL), stable mode-locking (SML) and incomplete mode-locking (IML). The onset of incomplete mode-locking is determined, in time domain, when the pulse train is modulated by an irregular envelope and the pulses contain a multi-peaked structure [See Fig. 7(c)]. Experimentally, this multi-peaked structure manifests itself in a coherence spike on top of a pedestal in the autocorrelation function. Two possible scenarios have been identified for this instability ${ }^{24}$ : For narrow bandwidth, a perturbation grows before the leading edge of the pulse due to an excessive gain, whereas for wide bandwidths the instability strongly depends on the cavity dispersion and self-phase modulation. We also find a narrow region of optical bistability for currents close-to-threshold and large reverse bias on the absorber. The behavior of the output power when the injection current is increased and subsequently decreased, as shown in Fig. 4, demonstrates that bistability leads to a small hysteresis cycle. The size of the hysteresis cycles depends on the level of unsaturated losses and the carriers lifetime. ${ }^{25}$ The slope of the $L-I$ curves decrease with the reverse bias due to the decrease in quantum efficiency due to the higher losses.

The increase of reverse bias provides pulse shortening through two mechanisms [Fig. 3(b)]: i) the decrease in saturation energy of the absorber, and ii) the faster recovery of the absorber. We find that self-phase modulation (SPM) increases for operation far from threshold, leading to large values of the time-bandwidth product (TBWP). Thus, the optimum situation is likely to occur close-to-threshold and for large reverse bias, as found experimentally. ${ }^{12}$ Pulsewidth investigations reveal a joint interplay of three different mechanisms, namely, pulse 
shaping, self-phase modulation, and gain saturation due to fast dynamics. The first mechanism relies on a necessary condition for pulse formation, i.e., the larger saturation energy in the amplifier than in the absorber. The second is the pulse chirp that originates from the dynamic changes in refractive index and because of the dispersion of the diffraction grating. As a consequence, the pulses broaden and the resulting TBWP is slightly above the transform-limited value. Finally, the fast effects included in the material dynamics, spectral hole burning and carrier-heating, provide a pulsewidth dependent contribution to the saturation energies ${ }^{26}$ when the pulsewidth $(\sim 1 \mathrm{ps})$ is an intermediate time scale between the stimulated carrier lifetime and the ultrafast processes.

The extent of the dynamical instabilities must be reduced to make the device sufficiently stable over a wide parameter range. There exists a large number of possible combinations of parameters, which make the optimization work cumbersome. Here, we concentrate in describing the metamorphosis of the phase-diagrams when the properties of the diffraction grating are varied.

Diffraction grating position. The relative position of the diffraction grating with respect to the gain and absorption curves is changed from $1.55 \mu \mathrm{m}$ to $1.515 \mu \mathrm{m}$ in Fig. 5. In the latter case, we find a strong tendency to Q-switching. The presence of Q-switching can be understood as the enhanced unsaturated losses introduced by the saturable absorber when the wavelength is close to the absorption edge and due to the longer carrier lifetime resulting from the lower threshold. At the onset of Q-switching, relaxation oscillations become undamped and the pulse train is modulated by a low frequency envelope. An interesting observation is that the Q-switching region reduces when the ratio $T_{R} / \tau_{e}$ increases, $T_{R}$ being the repetition period and $\tau_{e}$ the carrier lifetime. The lower saturation energy leads to a faster increase in unsaturated gain with injection current, thus favoring the
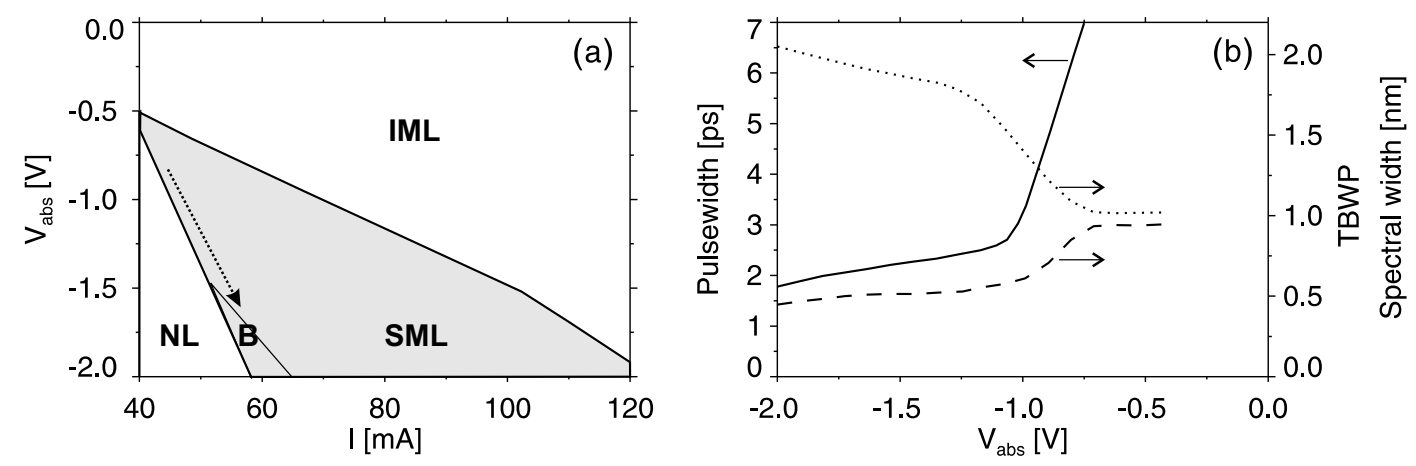

Figure 3. (a) Phase diagram in the absorber voltage versus injection current plane. The meaning of the symbols is $\mathrm{NL}=$ no laser emission, $\mathrm{SML}=$ stable mode-locking, $\mathrm{B}=$ Bistability, IML=incomplete mode-locking. (b) Uncompressed pulsewidth (solid), spectral width (dotted) and time-bandwidth product (dashed) when moving along the arrow in panel (a). Conditions $\lambda_{>}=1.55 \mu \mathrm{m}, \Delta \lambda_{B}=8 \mathrm{~nm}$, and $D_{\text {grat }}=0$.

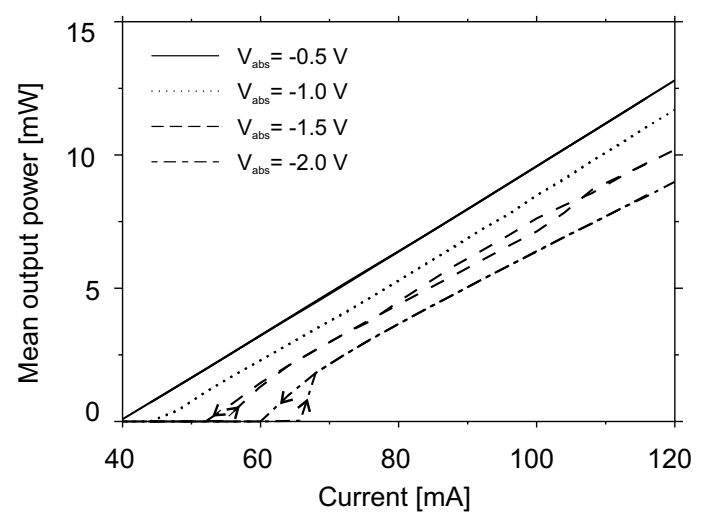

Figure 4. Light-current characteristics for different reverse bias on the absorber. The output power is measured at the absorber facet. Conditions $\lambda_{>}=1.55 \mu \mathrm{m}, \Delta \lambda_{B}=8 \mathrm{~nm}$, and $D_{\text {grat }}=0$.

$5 \quad$ Proc. SPIE 5452-70 (2004) 

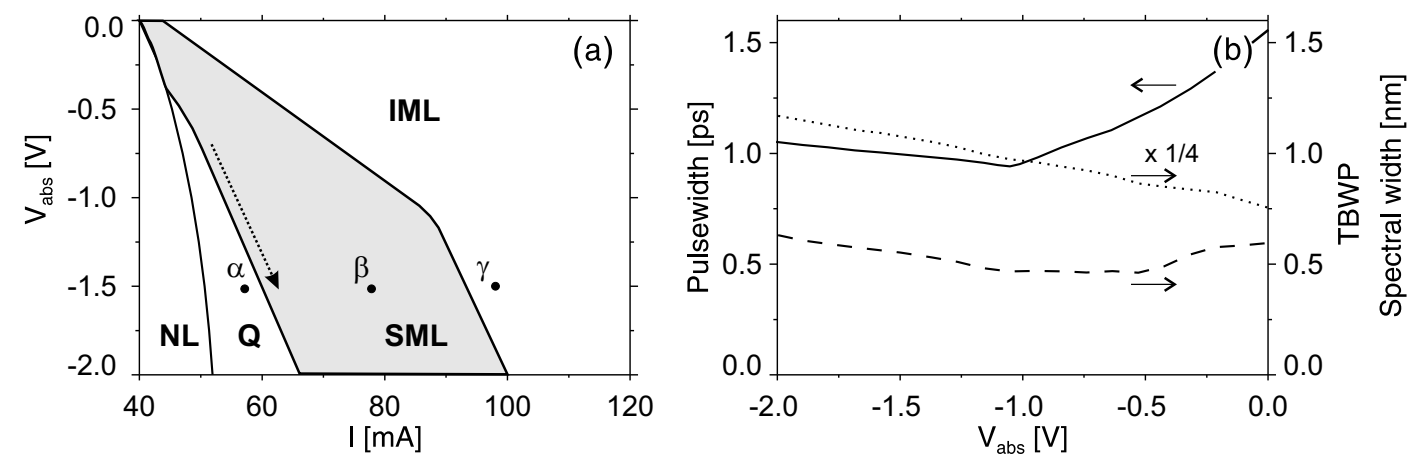

Figure 5. (a) Phase diagram in the absorber voltage versus injection current plane. The meaning of the symbols is $\mathrm{NL}=$ no laser emission, SML=stable mode-locking, $\mathrm{Q}=\mathrm{Q}$-switched mode-locking, IML=incomplete mode-locking. (b) Uncompressed pulsewidth (solid), spectral width reduced by a factor 4 (dotted) and time-bandwidth product (dashed) when moving along the arrow in panel (a). For the same conditions that in Fig. 3 except for $\lambda_{<}=1.515 \mu \mathrm{m}$.

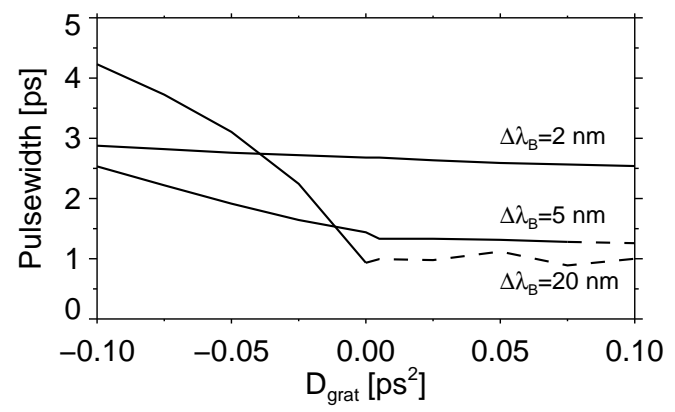

Figure 6. Effect of the grating bandwidth $\Delta \lambda_{B}$ and dispersion $D_{\text {grat }}$ on the stability and uncompressed pulsewidth. The solid (dashed) lines correspond to stable (unstable) mode-locking. Driving conditions: $I=70 \mathrm{~mA}, V_{a b s}=-2 \mathrm{~V}$, $\lambda=1.515 \mu \mathrm{m}$.

incomplete mode-locking instability. When moving to shorter wavelengths the ratio of saturation energies for the amplifier and the absorber, $s=E_{s a t, g} / E_{s a t, a}$ has a weak dependence on reverse bias, therefore we do not observe big changes in pulsewidth in Fig. $5(\mathrm{~b})$. The advantage of this driving condition is that the alpha-factors are smaller, reducing SPM and in turn the TBWP. Moreover, the optimum situation occurs at bias around -1 $\mathrm{V}$ since we have to avoid the Q-switching region. For higher reverse bias, the laser has to be driven far from the mode-locking threshold, and thus SPM starts to broaden the pulses again.

Diffraction grating bandwidth and chirp. In Fig. 6, we analyze the behavior around zero grating dispersion when the bandwidth is increased. The range of unstable mode-locking, denoted by dashed lines in the figure, increases when taking positive grating dispersion, and specially for wide filters. In the absence of grating dispersion, the pulses are blue-chirped due to the unbalanced SPM in the amplifier and absorber sections. When $D_{\text {grat }}<0$, the grating introduces an extra blue-chip that further broaden the pulses. The grating operates in the opposite direction when $D_{\text {grat }}>0$, i.e., compensating the initial blue chirp and, in turn, shortening the pulses. The pulse shortening, caused either by bandwidth enhancement or chirp compensation, results in a clear tendency to incomplete mode-locking. We have found that fast effects play a role in this instability, specially carrier heating which relaxation occurs at the pulsewidth time-scales.

There, of course, exists many other possible combinations of parameters. As already discussed, small unsaturated losses are preferable to prevent the onset of Q-switching. The different sources of losses, including internal losses, unsaturated absorption, and mirror losses should be minimized for obtaining low threshold currents. Low threshold currents decrease the ASE levels and in turn reduces the timing jitter. The active region of the present 

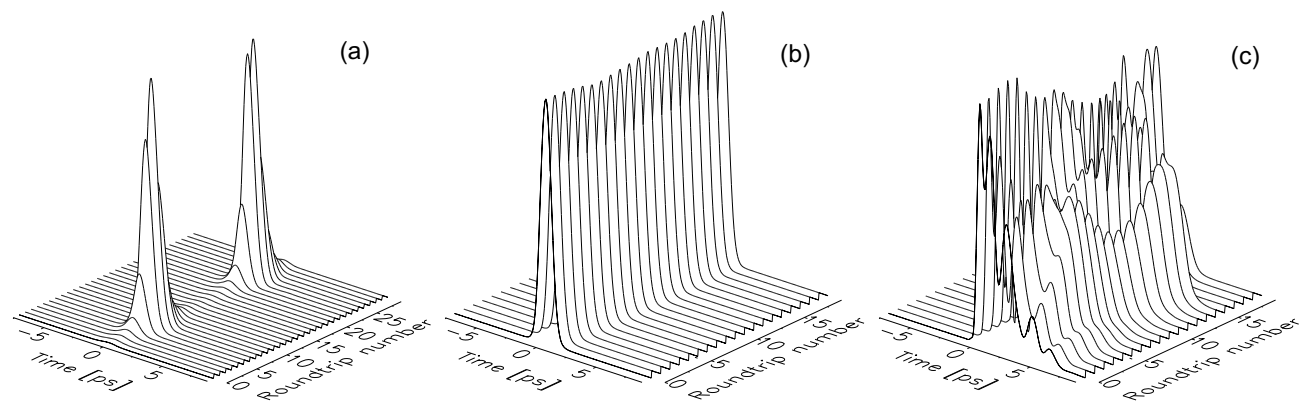

Figure 7. Evolution of the pulse for $V_{a b s}=-1.5 \mathrm{~V}$ in Fig. 5. (a) Fully-developed Q-switched mode-locking $I=58 \mathrm{~mA}$, (b) stable mode-locking $I=80 \mathrm{~mA}$, and (c) incomplete mode-locking $I=110 \mathrm{~mA}$.
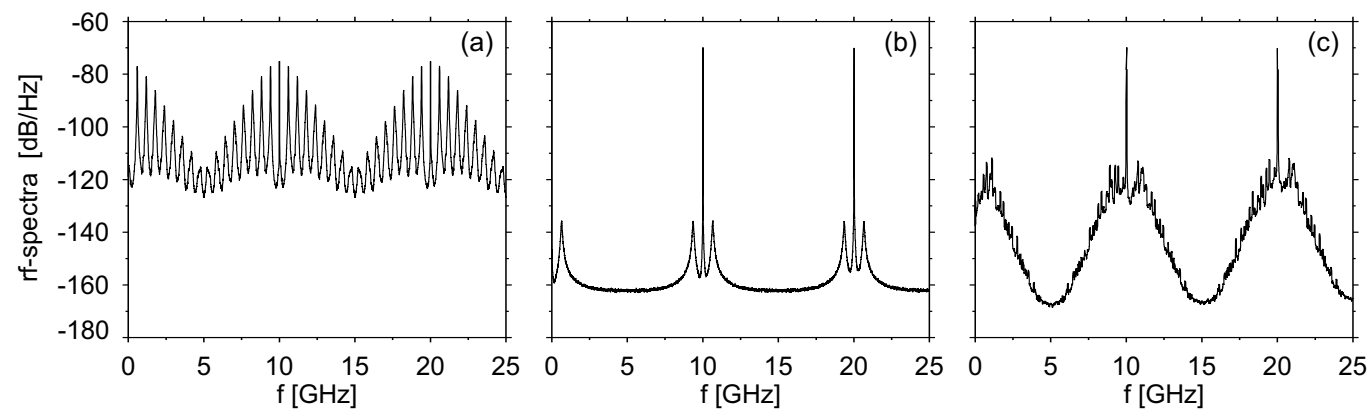

Figure 8. RF-spectra for the three situations in Fig. 7. (a) Fully-developed Q-switched mode-locking, (b) stable modelocking, and (c) incomplete mode-locking.

laser is composed by six QWs. If the number of wells is reduced, the optical confinement factor reduces leading to higher inversion and larger saturation energy. This case is particularly interesting because it provides high-power and low timing-jitter mode-locked semiconductor lasers. ${ }^{27}$

\section{TIMING JITTER}

In order to complete the characterization of the device, the dependence of timing jitter with respect to variation of the control parameters is investigated. The rf-spectrum, that is easily accessible in the experiments, can provide valuable information about the dynamics in the different regimes described in the preceding section. In Fig. 8, we plot the rf-spectra corresponding to the operation points $\alpha, \beta, \gamma$ annotated in the phase-diagram of Fig. 5. The signature of Q-switching can be easily identified in the rf-spectrum as the emergence of multiple peaks between two consecutive dominant harmonic peaks. On the other hand, the onset of incomplete modelocking can be identified by the sudden increase in amplitude noise around the harmonic peaks. RF-spectra can be used to quantify timing jitter by means of the von der Linde method ${ }^{28}$; see e.g. reference. ${ }^{29}$ In general, amplitude jitter and timing jitter are distributed in the rf-spectrum in different frequency intervals, thus the von der Linde method requires integration of noise skirts around successive harmonic peaks in order to separate timing jitter and amplitude jitter contributions. For convenience, we use an alternative, although equivalent, method to determine timing jitter. Our method establishes an analogy between the perturbation theory of the MEML $^{9,10}$ and our fully-distributed time-domain model. The timing fluctuations of a pulse train, $\Delta t_{n}$, are numerically determined with the pulse detection technique described in Appendix A. As we will show next, timing jitter can be fully determined from this quantity.

\subsection{Timing trajectories and timing variance}

We investigate the evolution of the timing fluctuation $\Delta t_{n}$ defined in Appendix A. In Fig. 9(a) we plot eight different realizations of the timing fluctuation, that correspond to different sequences of spontaneous emission

$7 \quad$ Proc. SPIE 5452-70 (2004) 
noise, all measured with respect to the same reference clock. The timing variance as function of the roundtrip number is obtained by performing the ensemble averaging of 20 series. The result is shown in Fig. 9(b), and it turns out that 20 realizations are still insufficient to completely smooth the trace. Notwithstanding, we can observe a linear increase in variance, similarly to what happens in a random walk process. ${ }^{30}$ The variance is unbounded at long times, as expected from the absence of any time reference in passive mode-locking. From the slope of the least squares line, we can estimate the kick size acting per roundtrip on the pulse to be $\sqrt{\sigma_{N}^{2} / N} \sim 5$ $\mathrm{fs}$, being in agreement with typical experimental values and predictions from the MEML. ${ }^{9}$ Small pulse-to-pulse timing jitter is required when using mode-locked lasers in optical sampling and data conversion applications. We have found that the kick size of the random walk reduces with the reverse bias, as shown in the inset of the Fig. 9(b). This dependence can be understood using the MEML ${ }^{9}$

$$
\sqrt{\sigma_{N}^{2} / N} \sim \frac{\tau}{\sqrt{E}}
$$

for large $N$. Hence if we take the pulse energy $E$ as constant, the kick size reduces with reverse bias because of the reduction in pulsewidth $\tau$.

The phase-noise spectrum is determined from the timing realizations through Eqs. (10)-(11). The phase-noise spectrum displays a linear increase when approaching to the harmonic peak as consequence of the asymptotic behavior of the variance for long times [Fig. 10(a)]. This is the typical $1 / \Omega^{2}$ spectrum characteristic of a random walk. We use the phase-noise spectrum to determine the timing jitter in a frequency range of interest. The minimum frequency resolution that allows for a statistical analysis with reasonable integration times corresponds to $\sim 300 \mathrm{kHz}$. Although timing jitter below this value is always present, it can be corrected using electronics. We use Eq. (12) to determine the timing jitter accumulated from $300 \mathrm{kHz}$ and beyond, up to the Nyquist frequency at $5 \mathrm{GHz}$. The integrated timing jitter increases before reaching a saturation value that corresponds to the root-mean-square (rms) timing jitter. Note however that, in passive mode-locking, the rms timing jitter would increase when taking finner frequency resolution. The result, shown in Fig. 10(b), indicates that frequencies below $10 \mathrm{MHz}$ provide the dominant contribution to timing jitter, thus justifying the integration interval [100 $\mathrm{Hz}, 10 \mathrm{MHz}]$ taken in experiments. ${ }^{22}$

From the above results we can conclude that passive mode-locking displays large timing jitter at low frequencies. In order to reduce the timing jitter of the ECMLL, one employs a modulation of the reverse bias (hybrid mode-locking). We consider a modulation as

$$
V_{a b s}(t)=V_{a b s}+\Delta V_{a b s} \cos \left(2 \pi f_{m} t\right) .
$$

The electrical noise from the synthesizer is neglected here, since it starts to be important only below $100 \mathrm{kHz}$. It is interesting to determine the synchronization locking range as function of the modulation strength $\Delta V_{a b s}$.
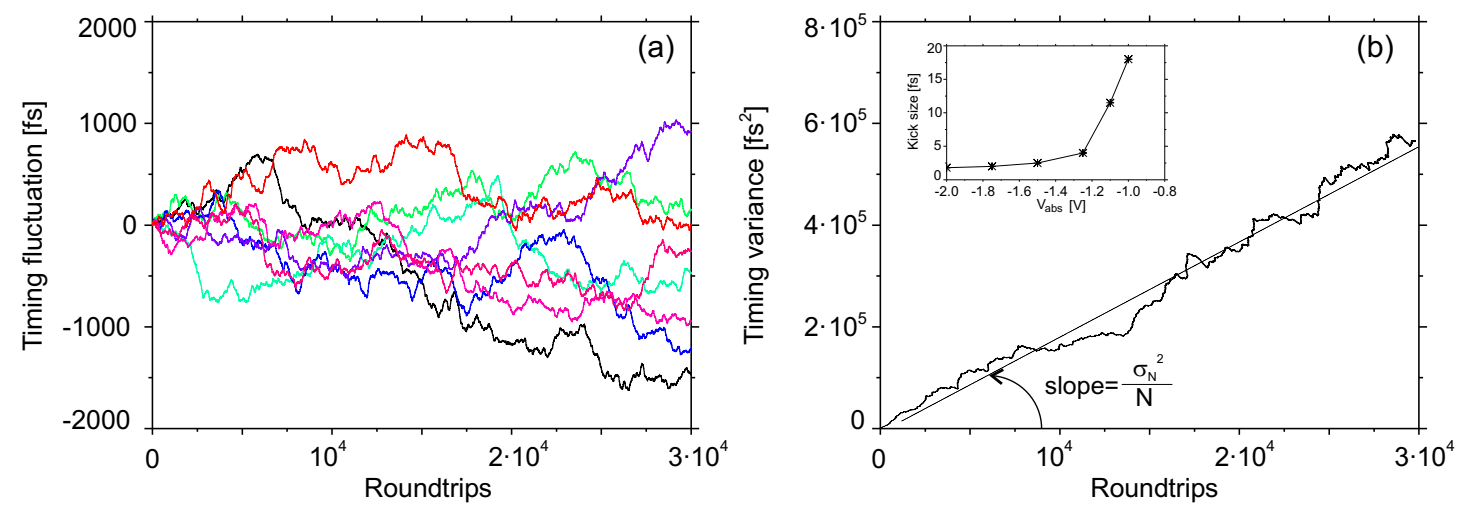

Figure 9. Passive mode-locking. (a) Eight realizations of the timing fluctuation corresponding to different sequences of the spontaneous emission. (b) Linear increase in timing variance characteristics of a random-walk process. Parameters: $V_{a b s}=-1.5 \mathrm{~V}$ and $I=60 \mathrm{~mA}$. 

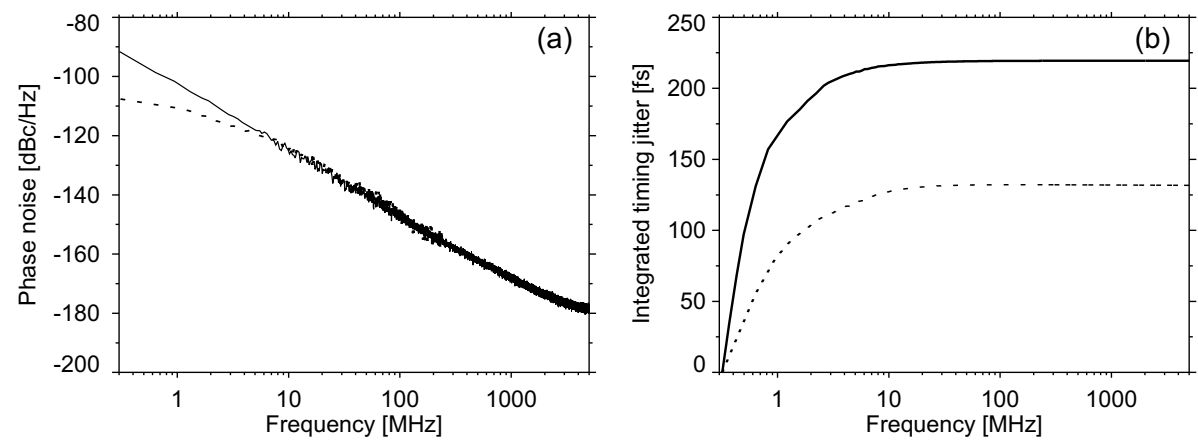

Figure 10. Comparison of timing jitter for passive mode-locking (solid curves) and hybrid-mode locking (dashed lines). (a) Phase noise spectrum [Eq. (11)] and integrated timing jitter [Eq. (12)]. Parameters: $V_{a b s}=-1 \mathrm{~V}, I=65 \mathrm{~mA}$, $\Delta V_{a b s}=0.25 \mathrm{~V}$ and $f_{m}=10.005299 \mathrm{GHz}$. The pulsewidth without modulation is $2.63 \mathrm{ps}$, and with modulation it is 2.85 ps.

However, this issue is out of the scope of this paper. We instead consider that $f_{m}$ is close to the passive modelocking frequency and always within the synchronization locking range. As a result of the modulation, the phase noise, depicted in dashed lines in Fig. 10, deviates from the straight line for frequencies below $10 \mathrm{MHz}$. This rolloff characteristic frequency depends on the strength of the modulation and the pulsewidth. ${ }^{31}$ The accumulated timing jitter is reduced owing to the reduction in phase noise. We find however a slight increase in pulsewidth owing to an extra chirp induced by the modulation.

\subsection{Dependence of timing jitter on driving conditions}

So far we have provided a practical example of the method implemented to determine the timing jitter in passive and hybrid mode-locking. Next, we make use this method to determine the variation of the timing jitter in the phase-diagrams of Sec. 3. Among the several situations described, we concentrate with the one described in Fig. 3 because it combines relative short pulsewidths with wide stability regions. Furthermore, the larger saturation energy of the amplifier induces smaller changes in carrier-density during a roundtrip and thus we expect better timing jitter performance.

Fig. 11 shows the evolution of timing jitter when the reverse bias and injection current are simultaneously varied close to the mode-locking threshold. The rms timing jitter decreases considerable when increasing the reverse bias, although the pulsewidth decreases as well. This observation may be related to the shorter pulse-topulse kick size of the random walk for higher reverse bias. This fact confirms that the optimum driving conditions takes place close to the mode-locking threshold and for high reverse bias. When a modulation is applied, at the passive frequency and $\Delta V_{a b s}=0.25 \mathrm{~V}$, the timing jitter is considerably reduced. The relative reduction is more noticeable for low reverse bias.

There exist a number of physical mechanisms contributing to timing jitter in passive mode-locked lasers. ${ }^{32}$ We restrict ourselves to briefly recall some of them. For example, the gain depletion in the amplifier can induce a net time shift of the pulse owing to the leading and trailing edges experience different amplification. ${ }^{11}$ We note that the amplifier and absorber sections tend to operate in opposite way. On the slow time scale, there exist at least two ways in which carrier density variations couple to timing jitter. Carrier-density variations induce a change in the index of refraction, that in turn induces a slight variation in emission frequency, and finally a change in group velocity due to group velocity dispersion (similar to Gordon-Haus jitter). The second mechanism is based, again on gain saturation, but now relies on the direct variations in group velocity with carrier density. A change in group velocity will provide slightly different roundtrip propagation times, hence introducing timing jitter. 


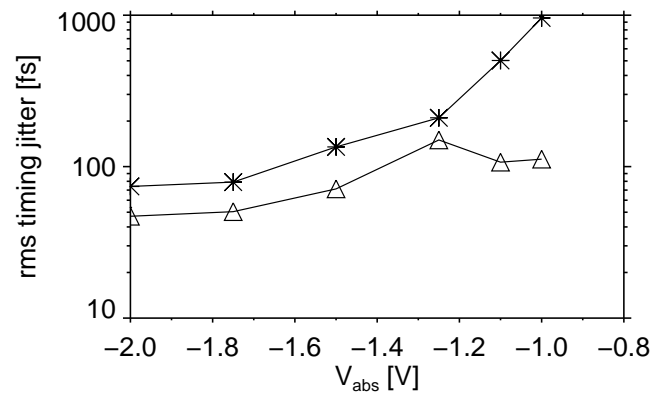

Figure 11. Evolution of the rms-timing jitter following the arrow in Fig. 3. Passive mode-locking (stars) and hybrid mode-locking (triangles).

\section{CONCLUSIONS}

A detailed theoretical description of an external-cavity mode-locked laser has been developed. The approach describes the contribution of gain saturation arising from ultrafast dynamics. The model has been used to characterize the mode-locking performance: identification of the limiting factors to the shortest pulsewidth and the timing jitter.

Concerning the first point, we found that the pulsewidth results from a joint interplay of fast and slow saturation effects together with self-phase modulation. A thorough understanding of this complex relationship can be used for obtaining shorter pulses and extending the regime of stable mode-locking. We have mentioned some strategies to avoid dynamical instabilities that affect the mode-locking quality and consequently the performance of the system.

In the second part, we have used the ECMLL model to investigate the timing jitter when the laser operates within a regime of stable mode-locking. It has been demonstrated that the timing variance in passive modelocking increases linearly with time, similarly to what happens in a random walk process. From the slope of the timing variance, we have estimated the kick size per roundtrip. The results indicate that the pulse-to-pulse kick size is generally small (1-20 fs), despite the fact that the rms timing jitter displays larger values (50$1000 \mathrm{fs}$ ). Timing jitter is reduced by nearly a factor of two, when applying a rf-modulation to the reverse-bias of the absorber. Our model correctly describes the dependence of pulsewidth and timing jitter over a wide parameter range. The shortest pulsewidth with optimum timing jitter takes place for currents close-to-threshold and large reverse bias. From the numerical point of view, the determination of timing jitter below $300 \mathrm{kHz}$ would require huge computational resources. Although the low frequency jitter is always present, it is not very relevant from the systems point of view because it can be corrected using electronics. The fully-distributed timedomain description could be used for analyzing the effect of several physical mechanisms introducing timing jitter, namely, net time shifts due to gain depletion, changes in group velocity initiated by emission-frequency fluctuations, carrier-induced group-velocity, etc. The understanding and quantification of these sources of timing jitter deserves further investigation.

\section{APPENDIX A.}

The pulse detection technique is implemented to numerically determine the timing jitter. A run time script looks for maxima above a predefined threshold, and determines the peak of each pulse. Once a peak is found, the program integrates the pulse intensity and the time weighted with the intensity, in order to determine the pulse energy and the mean pulse position

$$
U_{k}=\int_{t_{p, k}-T_{R} / 2}^{t_{p, k}+T_{R} / 2}\left|E_{\text {out }}(t)\right|^{2} d t, \quad \text { and } \quad t_{k}=\frac{1}{U_{k}} \int_{t_{p, k}-T_{R} / 2}^{t_{p, k}+T_{R} / 2}\left|E_{\text {out }}(t)\right|^{2} t d t
$$

$t_{p, k}$ being the time where the peak maximum was found, $E_{\text {out }}(t)$ the electric field at the output (absorber) facet, and $T_{R}$ an approximated repetition time. 
The deviation of the pulse train from an ideal clock with repetition $T_{C}$ defines the timing fluctuation $\Delta t_{k}=$ $t_{k}-k T_{C}$. For passive mode-locking, we take $T_{C}$ as the average of all the pulse to pulse separations, which roughly corresponds to the round-trip propagation time of the cold cavity. Note that the same clock has to be used for all the noise realizations. For hybrid mode-locking we simply take $T_{C}=1 / f_{m}, f_{m}$ the frequency of the external modulation. Timing jitter can be characterized from the timing fluctuation $\Delta t_{k}$. The evolution of the timing variance is obtained from

$$
\sigma_{\Delta t}^{2}\left(T_{k}\right)=\left\langle\left|\Delta t\left(T_{k}+T_{0}\right)-\Delta t\left(T_{0}\right)\right|^{2}\right\rangle
$$

with $T_{k}=k T_{C}$ and $\langle\cdot\rangle$ average over trajectories. The timing spectral density is numerically computed using $M$ noise realizations of the timing fluctuation

$$
\left\langle|\hat{\Delta} t(f)|^{2}\right\rangle \equiv \frac{1}{M} \sum_{j=1}^{M} \Delta T\left|\frac{1}{N} \sum_{k=1}^{N}\left\{\Delta t_{k}\right\}_{j} e^{-i 2 \pi f T_{k}}\right|^{2} .
$$

with $\Delta T=N T_{C}$ the time span. The massive data sets are Fourier transformed using the FFTW libraries. ${ }^{33}$ The phase noise spectrum reads

$$
\mathcal{L}(f)=\left(\frac{2 \pi}{T_{C}}\right)^{2}\left\langle|\hat{\Delta t}(f)|^{2}\right\rangle
$$

and the timing jitter integrated in a frequency range of interest is determined from

$$
\sigma_{\text {pc,t }}\left(f_{\text {low }}, f_{\text {high }}\right)=\frac{T_{C}}{2 \pi} \sqrt{\int_{f_{\text {low }}}^{f_{\text {high }}} 2 \mathcal{L}(f) d f} .
$$

\section{ACKNOWLEDGMENTS}

This work has been funded by the European project TOPRATE IST-2000-28657. The authors wish to thank fruitful discussions with K. Yvind, M. Kroh and B. Tromborg.

\section{REFERENCES}

1. H. Yokoyama, "Highly reliable mode-locked semiconductor lasers," IEICE Trans. Electron. E85-C, pp. 27$36,2002$.

2. E. A. Avrutin, J. H. Marsh, and E. L. Portnoi, "Monolithic and multi-GigaHertz mode-locked semiconductor lasers: Constructions, experiments, models and applications," IEE Proc.-Optoelectron. 147, pp. 251-278, 2000.

3. H. A. Haus, "Mode-locking of lasers," IEEE J. Selected Topics Quantum Electron. 6, pp. 1173-1185, 2000.

4. A. Gordon and B. Fischer, "Phase transition theory of many-mode ordering and pulse formation in lasers," Phys. Rev. Lett. 89, pp. 103901-3, 2002.

5. H. A. Haus, "Theory of mode locking with a fast saturable absorber," J. Appl. Phys. 46, pp. 3049-3058, 1975 .

6. H. A. Haus, "Modelocking of semiconductor laser diodes," Jap. J. Appl. Phys. 20, pp. 1007-1020, 1981.

7. J. C. Chen, H. A. Haus, and E. P. Ippen, "Stability of lasers mode locked by two saturable absorbers," IEEE J. Quantum Electron. 29, pp. 1228-1232, 1993.

8. J. A. Leegwater, "Theory of mode-locked semiconductor lasers," IEEE J. Quantum Electron. 32, pp. 17821790, 1996.

9. L. A. Jiang, M. E. Grein, H. A. Haus, and E. P. Ippen, "Noise of mode-locked semiconductor lasers," IEEE J. Selected Topics Quantum Electron. 7, pp. 159-167, 2001.

10. H. A. Haus and A. Mecozzi, "Noise of mode-locked lasers," IEEE J. Quantum Electron. 29, pp. 983-996, 1993. 
11. G. P. Agrawal and N. A. Olsson, "Self-phase modulation and spectral broadening of optical pulses in semiconductor laser amplifiers," IEEE J. Quantum Electron. 25, pp. 2297-2306, 1989.

12. K. Yvind, P. M. W. Skovgaard, J. Moerk, J. Hanberg, and M. Kroh, "Performance of external cavity mode-locked semiconductor lasers employing reverse biased saturable absorbers," Physica Scripta T101, pp. 129-132, 2002.

13. M. Shtaif, B. Tromborg, and G. Eisenstein, "Noise spectra of semiconductor optical amplifiers: relation between semiclassical and quantum descriptions," IEEE J. Quantum Electron. 34, pp. 869-878, 1998.

14. J. Moerk, M. Willatzen, J. Mark, M. Svendsen, and C. P. Seltzer, "Characterization and modelling of ultrafast carrier dynamics in quantum well optical amplifiers," Proc. SPIE 2146, pp. 52-67, 1994.

15. J. Moerk and J. Mark, "Time-resolved spectroscopy of semiconductor laser devices: Experiments and modelling," Proc. SPIE 2399, pp. 146-157, 1995.

16. S. Bischoff, J. Moerk, T. Franck, S. D. Brorson, M. Hofmann, K. Fröjdh, L. Prip, and M. P. Soerensen, "Monolithic colliding pulse mode-locked semiconductor lasers," Quantum Semiclass. Opt. 9, pp. 655-674, 1997.

17. D. C. Hutchings, M. Sheik-Bahae, D. J. Hagan, and E. W. van Stryland, "Kramers-krönig relations in nolinear optics," Opt. and Quantum Electron. 24, pp. 1-30, 1992.

18. S. Bischoff, Modelling colliding-pulse mode-locked semiconductor lasers, $\mathrm{PhD}$ dissertation, Institut for Matematisk Modellering, Lyngby, Denmark, 1997.

19. J. R. Karin, R. J. Helkey, D. J. Derickson, R. Nagarajan, D. S. Allin, J. E. Bowers, and R. L. Thornton, "Ultrafast dynamics in field-enhanced saturable absorbers," Appl. Phys. Lett. 64, pp. 676-678, 1994.

20. P. J. Stevens, M. Whitehead, G. Parry, and K. Woodbridge, "Computer modeling of the electric field dependent absorption spectrum of multiple quantum well material," IEEE J. Quantum Electron. 24, pp. 20072016, 1988.

21. R. Toral and A. Chakrabarti, "Generation of Gaussian distributed random numbers by using a numerical inversion method," Comp. Phys. Commun. 74, p. 327, 1993.

22. M. Kroh et al. This device has been fabricated and characterized at Heinrich-Hertz-Institute, Berlin, Germany.

23. D. J. Derickson, R. J. Helkey, A. Mar, J. R. Karin, J. E. Bowers, and R. L. Thornton, "Supression of multiple pulse formation in external-cavity mode-locked semiconductor lasers using intrawaveguide saturable absorbers," IEEE Photon. Technol. Lett. 4, pp. 333-335, 1992.

24. M. Schell, M. Tsuchiya, and T. Kamiya, "Chirp and stability of mode-locked semiconductor lasers," IEEE J. Quantum Electron. 32, pp. 1180-1190, 1996.

25. H. Kawaguchi, "Optical bistability and chaos in a semiconductor laser with a saturable absorber," Appl. Phys. Lett. 45, pp. 1264-1266, 1984.

26. A. Mecozzi and J. Moerk, "Saturation effects in nondegenerate four-wave mixing between short optical pulses in semiconductor laser amplifiers," IEEE J. Selected Topics Quantum Electron. 3, pp. 1190-1207, 1997.

27. K. Yvind, D. Larsson, L. J. Christiansen, C. Angelo, L. K. Oxenloewe, J. Moerk, D. Birkedal, J. H. Hvam, and J. Hanberg, "Low-jitter and high-power all-active mode-locked lasers," In proceedings of the conference ECOC Rimini, 2003.

28. D. von der Linde, "Characterization of the noise in continuously operating mode-locked lasers," Appl. Phys. B 39, pp. 201-217, 1986.

29. B. Zhu, R. V. Penty, A. Wonfor, E. Lach, and H. D. Summers, "Theoretical analysis of timing jitter in monolithic multisection mode-locked dbr laser diodes," IEEE J. Quantum Electron. 33, pp. 1216-1220, 1997.

30. M. San Miguel and R. Toral, "Stochastic effects in physical systems," Procs. of instabilities and nonequilibrium structures VI, p. 35, Kluwer academic, Dordrecht, 2000.

31. L. A. Jiang, M. E. Grein, J. K. Chandalia, E. P. Ippen, and H. Yokoyama, "Retiming dynamics of modelocked semiconductor lasers," Electron. Lett. 38, pp. 1446-1447, 2002.

32. L. A. Jiang, Ultralow-noise modelocked lasers, PhD dissertation MIT, Massachusetts, 2002.

33. M. Frigo and S. G. Johnson, "The fastest Fourier transform in the west (FFTW), release 3.0.1," http://www.fftw.org . 